\title{
STABILITY ANALYSIS OF NONLINEAR TIME-DELAYED SYSTEMS WITH APPLICATION TO BIOLOGICAL MODELS
}

\author{
H.A. KRUTHIKA ${ }^{a}$, ARUN D. MAHINDRAKAR ${ }^{a, *}$, RAMKRISHNA PASUMARTHY $^{a}$ \\ ${ }^{a}$ Department of Electrical Engineering \\ Indian Institute of Technology Madras, Chennai, India \\ e-mail: kruthika.ha@gmail.com, \{arun_dm, ramkrishna\}@iitm.ac.in
}

\begin{abstract}
In this paper, we analyse the local stability of a gene-regulatory network and immunotherapy for cancer modelled as nonlinear time-delay systems. A numerically generated kernel, using the sum-of-squares decomposition of multivariate polynomials, is used in the construction of an appropriate Lyapunov-Krasovskii functional for stability analysis of the networks around an equilibrium point. This analysis translates to verifying equivalent LMI conditions. A delay-independent asymptotic stability of a second-order model of a gene regulatory network, taking into consideration multiple commensurate delays, is established. In the case of cancer immunotherapy, a predator-prey type model is adopted to describe the dynamics with cancer cells and immune cells contributing to the predator-prey population, respectively. A delay-dependent asymptotic stability of the cancer-free equilibrium point is proved. Apart from the system and control point of view, in the case of gene-regulatory networks such stability analysis of dynamics aids mimicking gene networks synthetically using integrated circuits like neurochips learnt from biological neural networks, and in the case of cancer immunotherapy it helps determine the long-term outcome of therapy and thus aids oncologists in deciding upon the right approach.
\end{abstract}

Keywords: time-delay, cancer immunotherapy, gene-regulatory network, sum of squares.

\section{Introduction}

Applications of control in biology and biochemical systems have a rich history. The emerging interdisciplinary field of systems biology emphasizes the role of control theory in understanding the complex interactions within biological systems. This concept has been used widely in biosciences in a variety of contexts. In this paper, we focus on two such applications: (i) gene regulatory networks, which are a set of genes, or parts of genes, that interact with each other to control a specific cell function (Bernot et al., 2013; Chen and Aihara, 2002); (ii) the cancer immunotherapy model (Andrew et al., 2007; Babbs, 2011; d'Onofrio, 2005), where the human immune system is used to treat cancer by provoking the immune system to attack tumor cells using cancer antigens as targets. Towards better understanding of the complex behaviour of such applications, we need to develop mathematical models and, in many cases, help predict the corresponding experimental observations.

One of the common modeling techniques is the use

${ }^{*}$ Corresponding author of ordinary differential equations (ODEs). However, in cases involving gene transcription and the cancer immunotherapy model, the processes involve several reactions which may not be accurately modelled as instantaneous reactions. To account for the delay in time taken for the completion of an entire process, it was proposed to model these reactions as single step multiple-delayed reactions. Mathematically, this would mean that the models would be ODEs associated with time-delays, which is in the control parlance referred to as time delay systems (Kolmanovskii and Myshkis, 1999; Gu et al., 2003), or in maths_-a delay differential equation.

A second-order predator-prey model that accounts for the process time-delays in protein synthesis is considered for nonlinear stability analysis, while third-order dynamics with a single delay are used for the immunotherapy model. Predator-prey models that incorporate a much involved behaviour of the species can be found in the work of She and Li (2013). From the systems point of view, these systems can either be linear or nonlinear in nature. Several results have appeared in the literature with regard to studying stability 
of linear systems with time delays (Richard, 2003; Gu et al., 2003; Kolmanovskii and Myshkis, 1999). The Mikhailov criterion in the frequency domain uses the principle of argument, while integral quadratic constraints (IQCs) employ also a frequency domain approach (Kao and Rantzer, 2007). For nonlinear systems, there are only a handful of results, such as the construction of a Lyapunov-Krasovskii (L-K) functional (Papachristodoulou, 2004; Pasumarthy and Kao, 2009; Kao and Pasumarthy, 2012; Mazenc and Niculescu, 2001). Our approach here is the sum-of-squares method (Papachristodoulou and Prajna, 2005), which will be our basis for generating results in this paper. Other approaches to stability analysis exploit the system structure. Bodnar (2015) adopts the method developed by Eduardo and Ruiz-Herrera (2013) to determine the global stability of an equilibrium solution of a cascade of chemical reactions with feedback by investigating the asymptotic behaviour of some corresponding discrete dynamical system. In the works of Bo et al. (2012), Zhong et al. (2014), or Liu et al. (2015; 2016a), Liu et al. (2016b), several results pertaining to the global stability analysis of neural networks with a time-delay can be found.

The main contributions of this paper are the following. A numerically generated kernel is used in the Lyapunov-Krasovskii functional to establish delay-independent asymptotic stability of the equilibrium of interest in the case of the gene-regulatory model. In the case of the immunotherapy model, the equilibrium point of interest is shown to be delay-dependent, and the local uniformly asymptotic stability is established by numerically generating a kernel for a Lyapunov functional with a known structure. The process of constructing kernels using the SOS process forms the main theoretical tool; however, the method can be considered to be numerical owing to the numerical solution to the LMI conditions and SOS kernels, rather than an analytical one. Nonetheless, in both the examples, the process of constructing kernels using the SOS process is not straightforward as several iterations are made in arriving at the $\mathrm{L}-\mathrm{K}$ and Lyapunov functionals.

Apart from the system and control point of view, stability analysis of the dynamics of gene regulatory networks aids mimicking gene networks synthetically using integrated circuits like neurochips learnt from biological neural networks, and in the case of cancer immunotherapy it helps determine the long-term outcome of therapy and thus helps oncologists to decide upon the right approach.

The rest of the paper is organized as follows. Section 2 describes the basic mechanism and model of the gene regulatory network followed by the main results concerning the nonlinear stability analysis and simulation results. In Section 3, the mechanism involving the triggering of immune cells and the eventual destruction of cancer cells is discussed. Results pertaining to stability form the core with simulations in the end. Biological implications of stability results are discussed in Section 4 with concluding remarks given in Section 5 .

1.1. Mathematical preliminaries. Let $\mathbb{R}^{n}$ be the $n$-dimensional real Euclidean space with the norm $|x|$ for $x \in \mathbb{R}^{n}$. We denote by $\mathbb{R}_{+}$and $\mathbb{R}_{++}$the set of nonnegative and positive real numbers, respectively. $\mathcal{C}\left([a, b], \mathbb{R}^{n}\right)$ denotes the Banach space of continuous functions that map the interval $[a, b]$, having $b>a$, into $\mathbb{R}^{n}$ with the topology of uniform convergence. For any $\phi \in \mathcal{C}\left([a, b], \mathbb{R}^{n}\right)$, the norm of $\phi$ is defined as $\|\phi\|=\sup _{a<\theta<b}|\phi(\theta)|$. For $\tau>0$ and any $b>0$, we let $\psi \in \mathcal{C}\left([-\tau, b], \mathbb{R}^{n}\right)$ be any continuous function of time. For any $t \in[-\tau, b]$ we denote by $\psi_{t}$ the segment of $\psi(t)$ defined by $\psi_{t}=\psi(t+\theta), \theta \in[-\tau, 0]$. A multivariate polynomial $p(x), x \in \mathbb{R}^{n}$, is an SOS if there exist polynomials $f_{i}(x), i=1, \ldots, M$, such that $p(x)=\sum_{i=1}^{M} f_{i}^{2}(x)$. A polynomial $p(x)$ of degree $2 d$ is an SOS if and only if there exist a positive semidefinite matrix $Q$ and a vector $Z(x)$ containing monomials in $x$ of degree $\leq d$ so that $p=Z(x)^{\top} Q Z(x)$ (Parrilo, 2000).

\section{Gene regulatory networks}

The basic structural and functional unit of living organisms is the cell. Cells by themselves are complex entities, be it a prokaryotic cell, which does not contain a nucleus, or a eukaryotic cell, which contains a nucleus. Of the numerous activities that a cell performs, protein synthesis is of prime importance. Proteins are required for the modulation and maintenance of cellular activities. Protein synthesis is a collection of various sequential processes which ultimately result in the production of the target protein in specific quantities. The ensemble of such sequential processes is also called gene regulation. The concept of regulation comes up in gene expression because genes are allowed to express themselves, resulting in functional products such as proteins only when needed. This is due to various biological reasons. Thus a gene regulation system consists of genes, cis-elements and regulators (Aluru, 2005). Though there are a number of sequential processes which ultimately result in the production of protein, transcription and translation are the prominent ones, which are also well understood. The sequence of events (see Fig. 1) which leads to protein synthesis (Bernot et al., 2013) is the following:

1. an external stimulus such as an environmental condition, a developmental stage of the organism, stress, diseases, etc.;

2. stimulation of a transcription factor; 
3. transcription of a particular gene;

4. production of mRNA (messenger-RNA);

5. translation of mRNA resulting in the production of protein.

Regulators are nothing but transcription factors, which are themselves proteins. These transcription factors bind themselves to cis-elements, which form the noncoding part of DNA. The cis-element is a short DNA sequence that is specific to a particular transcription factor and hence helps regulate the expression of a particular gene. The genes, regulators and the regulatory connections between them form gene regulatory networks (Aluru, 2005).

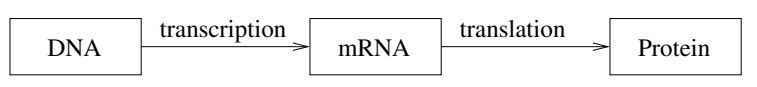

Fig. 1. Protein synthesis.

Two examples which illustrate how environmental conditions might trigger protein synthesis are those of lac operon in bacteria and hypoxia inducible factor- 1 $(H I F-1)$ in mammalian cells. The former refers to a bacterial genome and is composed of lacZ, lacY and lacA genes. When there is a lack of glucose and absence of lactose in the cell environment, catabolite activator protein favours the transcription of lac operon genes, allowing the consumption of lactose as a source of energy. In the absence of lactose, a lactose repressor protein impairs the transcription of lac operon genes.

The second example is the adaptation of mammalian cells to decrease oxygen pressure by stimulating the transcription factor HIF-1, which binds to the cis-elements, i.e., hypoxia response elements, which are DNA motifs associated with a series of genes involved in adaptation to low oxygen concentration. The gene which is well known for inducing red blood cells production is erythropoietin, which secretes the EPO protein and ultimately leads to an increase in erythropoiesis. Under the normal oxygen level concentrations, the stimulation of the epo gene is inhibited.

2.1. Mathematical modelling. Modelling approaches to gene regulatory networks can be roughly divided into continuous models and logical ones (Bernot et al., 2013). The choice depends upon the kind of data available and the objective. Different approaches followed until now include Boolean (Kauffman, 1969), discrete (Thomas, 1991), continuous differential (Goodwin, 1963) and piecewise affine (De Jong, 2002) models. Continuous models require quantitative biological data in order to fit the parameters but give a better picture of the dynamics of the system. Logical or Boolean models describe the state of genes in on/off mode along with logical links between them. Both the models can be used to study switching or oscillatory behaviours of gene networks. In this paper, we will be dealing only with the continuous model.

From a control point of view, a feedback exists in gene regulatory networks where the proteins produced due to a particular translation of mRNA may act as transcription factors for some other gene. When two proteins mutually repress or activate each other, this is known as a positive feedback loop. If one of them represses the other while the other activates it, then this is a negative feedback loop. Sharma et al. (2014) demonstrate the realization of fundamental logic operations and memory elements on delayed synthetic gene networks.

The process of protein synthesis is initiated with the transcription factors binding to the cis-elements of the genes, and then the transcription process starts. Other proteins may also bind to specific sites of genes to enhance or inhibit the transcription process. In the former case, the transcription factor is known as an activator, and in the latter as a repressor. The translation of mRNA produces the target protein. The law of mass action is used to arrive at the equation describing translation and transcription, with mRNA and protein concentrations as the variables. The generic equation which describes the rate of change in concentrations of $n$ molecular species $\left(x_{1}, x_{2}, \ldots, x_{n}\right)$ $\in \mathbb{R}_{+}^{n}$ is given by the difference between the production or synthesis of $i$-th molecular species and the degradation or transformation of the same into some other molecular species,

$$
\dot{x}_{i}=g_{i}(x)-d_{i}(x),
$$

where $g_{i}$ represents the $i$-th synthesis function and $d_{i}$ represents the $i$-th degradation function. A generic network model representing the dynamics of transcription and translation is given by (Chen and Aihara, 2002)

$$
\begin{aligned}
\dot{m}(t) & =-K_{m} m(t)+c\left(p\left(t, \tau_{p}\right)\right), \\
\dot{p}(t) & =-K_{p} p(t)+d\left(m\left(t, \tau_{m}\right)\right),
\end{aligned}
$$

where

$$
\begin{aligned}
p= & \text { concentrations of proteins }, \\
m= & \text { concentrations of mRNAs, } \\
K_{p}= & \text { degradation rates of proteins } \\
K_{m}= & \text { degradation rates of mRNAs }, \\
c\left(p\left(t, \tau_{p}\right)\right)= & \text { nonlinear function describing the } \\
& \text { synthesis/inhibition rate of mRNAs, } \\
d\left(m\left(t, \tau_{m}\right)\right)= & \text { nonlinear function describing the } \\
& \text { synthesis/inhibition rate of proteins, } \\
\tau_{p}= & \text { delay in the translation of proteins, } \\
\tau_{m}= & \text { delay in the transcription of mRNA, } \\
m= & \left(m_{1}, m_{2}, \ldots, m_{n}\right) \in \mathbb{R}_{+}^{n},
\end{aligned}
$$




$$
\begin{aligned}
p & =\left(p_{1}, p_{2}, \ldots, p_{n}\right) \in \mathbb{R}_{+}^{n}, \\
K_{m} & =\operatorname{diag}\left(k_{m 1}, \ldots, k_{m n}\right) \in \mathbb{R}_{+}^{n \times n}, \\
K_{p} & =\operatorname{diag}\left(k_{p 1}, \ldots, k_{p n}\right) \in \mathbb{R}_{+}^{n \times n}, \\
\tau_{m} & =\operatorname{diag}\left(\tau_{m 1}, \ldots, \tau_{m n}\right) \in \mathbb{R}_{+}^{n}, \\
\tau_{p} & =\operatorname{diag}\left(\tau_{p 1}, \ldots, \tau_{p n}\right) \in \mathbb{R}_{+}^{n}, \\
m\left(t, \tau_{m}\right) & =\left(m_{1}\left(t-\tau_{m 1}\right), \ldots, m_{n}\left(t-\tau_{m n}\right)\right), \\
p\left(t, \tau_{p}\right) & =\left(p_{1}\left(t-\tau_{p 1}\right), \ldots, p_{n}\left(t-\tau_{p n}\right)\right), \\
c(p) & =\left(c_{1}(p), \ldots, c_{n}(p)\right) \in \mathbb{R}_{+}^{n}, \\
d(m) & =\left(d_{1}\left(m_{1}\right), \ldots, d_{n}\left(m_{n}\right)\right) \in \mathbb{R}_{+}^{n} .
\end{aligned}
$$

A schematic representation of a feedback regulatory network (Chen and Aihara, 2002) is shown in Fig. 2

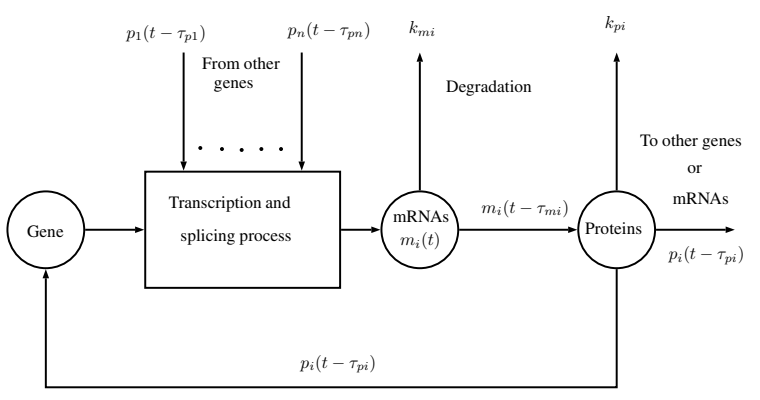

Fig. 2. Gene regulatory network with feedback.

Based on the same formalism, the model considered for further analysis is that of a negative feedback gene regulatory network, where the protein produced by the gene acts as a transcription factor and inhibits its own production. The dynamics (Loiseau et al., 2009, p. 137) are given by

$$
\begin{aligned}
& \dot{x}_{1}=-k_{1} x_{1}+a_{1} x_{2}\left(t-\tau_{1}\right), \\
& \dot{x}_{2}=-k_{2} x_{2}+\frac{a_{2}}{1+\left(x_{1}\left(t-\tau_{2}\right)\right)^{n}},
\end{aligned}
$$

where

$$
\begin{aligned}
& x_{1}=\text { concentration of protein, } \\
& x_{2}=\text { concentration of mRNA, } \\
& k_{1}=\text { degradation rate of protein, } \\
& k_{2}=\text { degradation rate of mRNA, } \\
& a_{1}=\text { translation rate, } \\
& a_{2}=\text { transcription rate, } \\
& \tau_{1}=\text { delay in translation, } \\
& \tau_{2}=\text { delay in transcription. }
\end{aligned}
$$

The synthesis function $g(x)$ in the case of an activator is given by $\frac{x^{n}}{\theta^{n}+x^{n}}$. This is also known as the Hill function, a monotonically increasing $\mathrm{S}$-shaped function. In the case of a repressor, it is $1-\frac{x^{n}}{\theta^{n}+x^{n}}$, i.e., $\frac{\theta^{n}}{\theta^{n}+x^{n}}$, a monotonically decreasing $\mathrm{S}$-shaped function. Here $\theta$ is the activation coefficient and $n$ is the Hill coefficient. The state variables $x_{i} \in \mathbb{R}_{+}, \tau_{i} \in \mathbb{R}_{+}, i=1,2$, and the parameters $k_{1}, k_{2}, a_{1}, a_{2} \in \mathbb{R}_{+}$. The starting point for further analysis would be to find the equilibrium points for the model described by (2). The case for which the Hill coefficient $n=1$ is considered.

With $n=1$, the dynamics given by (2) can be rewritten as

$$
\begin{aligned}
& \dot{x}_{1}(t)=-k_{1} x_{1}(t)+a_{1} x_{2}\left(t-\tau_{1}\right), \\
& \dot{x}_{2}(t)=-k_{2} x_{2}(t)+\frac{a_{2}}{1+x_{1}\left(t-\tau_{2}\right)} .
\end{aligned}
$$

2.2. Stability of time-delay systems. The system of equations (3) constitutes a delay differential equation, more generally written as

$$
\dot{x}(t)=f\left(x_{t}\right),
$$

where $f: \mathcal{C}\left([-\xi, 0], \mathbb{R}^{n}\right) \rightarrow \mathbb{R}^{n}$ and for $t>0 x_{t} \in$ $\mathcal{C}\left([-\xi, 0], \mathbb{R}^{n}\right)$ is defined as $x_{t}(\theta)=x(t+\theta)$ for $\theta \in$ $[-\xi, 0]$. The initial condition of (4) is denoted by $x_{t_{0}} \in$ $\mathcal{C}\left([-\xi, 0], \mathbb{R}^{n}\right)$. Let $\Omega \subset \mathcal{C}\left([-\xi, 0], \mathbb{R}^{n}\right)$ be open. If $f(\phi)$ is Lipschitz continuous in $\phi$ on every compact set $\Omega_{0} \subset \Omega$, then (4) has a unique solution through $x_{t 0} \in \Omega_{0}$. We assume that (4) has a unique solution for any initial condition $x_{t 0}$. With no loss of generality, we assume the trivial solution of (4) to be the equilibrium point of interest. The concept of the stability of the solution $x(t)=$ 0 is given below.

Definition 1. The solution $x(t)=0$ of (4) is called stable if for any $\epsilon>0$ there exists a $\delta(\epsilon)>0$ such that for any initial condition $x_{t_{0}}$ which satisfies $\left\|x_{t_{0}}\right\|<\delta(\epsilon)$ the corresponding solution $x(t)$ satisfies $|x(t)|<\epsilon$ for all $t \geq 0$. It is called asymptotically stable if it is stable and $\overline{\delta(\epsilon)}$ can be chosen such that $\left\|x_{t_{0}}\right\|<\delta(\epsilon)$ implies that $\lim _{t \rightarrow \infty} x(t) \rightarrow 0$. It is called globally asymptotically stable if it is asymptotically stable, and for any initial condition $x_{t_{0}}$ the corresponding solution $x(t)$ approaches to 0 as $t \rightarrow \infty$ no matter how large $\left\|x_{t_{0}}\right\|$ is.

A key tool for studying the stability property of systems governed by such equations is the Lyapunov-Krasovskii theorem, by which the stability criteria presented in this paper will be derived. The theorem is briefly summarized below.

Let $V: \mathcal{C}\left([-\xi, 0], \mathbb{R}^{n}\right) \rightarrow \mathbb{R}$. The derivative of $V$ with respect to time along the solution $x$ of (4) is

$$
\dot{V}\left(x_{t}\right)=\limsup _{\Delta t \rightarrow 0} \frac{1}{\Delta t}\left(V\left(x_{t+\Delta t}\right)-V\left(x_{t}\right)\right) .
$$

The following theorem ( $\mathrm{Gu}$ et al., 2003; Kolmanovskii and Myshkis, 1999) provides sufficient conditions for the stability of (4). 
Theorem 1. (Lyapunov-Krasovskii) Suppose that $f$ : $\mathcal{C}\left([-\xi, 0], \mathbb{R}^{n}\right) \longrightarrow \mathbb{R}^{n}$ in (4) maps bounded sets in $\mathcal{C}\left([-\xi, 0], \mathbb{R}^{n}\right)$ into bounded sets in $\mathbb{R}^{n}$, and that $u$, $v$, $w$ are continuous nonnegative nondecreasing functions, where $u$ and $v$ satisfy $u(0)=v(0)=0$, and $u(s)>0$ $v(s)>0$ for $s>0$. If there exists $V: \mathcal{C}\left([-\xi, 0], \mathbb{R}^{n}\right) \rightarrow$ $\mathbb{R}^{n}$ such that $V$ and the derivative of $V$ along the solution of (4) satisfy

$$
u(\|\phi(0)\|) \leq V(\phi) \leq v(\|\phi\|)
$$

and

$$
\dot{V}(\phi) \leq-w(\|\phi(0)\|)
$$

for all $\phi \in \mathcal{C}\left([-\xi, 0], \mathbb{R}^{n}\right)$, then the solution $x(t)=0$ of (4) is stable. If, in addition, $w(s)>0$ for any $s>0$, then the solution $x(t)=0$ of (4) is asymptotically stable. Finally, if $\lim _{s \rightarrow \infty} u(s)=\infty$, then we have the global asymptotic stability.

2.3. Local stability analysis. We analyze the stability of the system around an equilibrium point $x^{*}=\left(x_{1}^{*}, x_{2}^{*}\right)$, with $f\left(x^{*}\right)=0$. By defining $\tilde{x}=x-x^{*}$, linearizing the system (3) yields

$$
\begin{aligned}
\left(\begin{array}{c}
\dot{\tilde{x}}_{1}(t) \\
\dot{\tilde{x}}_{2}(t)
\end{array}\right)= & A_{0}\left(\begin{array}{c}
\tilde{x}_{1}(t) \\
\tilde{x}_{2}(t)
\end{array}\right)+A_{1}\left(\begin{array}{c}
\tilde{x}_{1}\left(t-\tau_{1}\right) \\
\tilde{x}_{2}\left(t-\tau_{1}\right)
\end{array}\right) \\
& +A_{2}\left(\begin{array}{c}
\tilde{x}_{1}\left(t-\tau_{2}\right) \\
\tilde{x}_{2}\left(t-\tau_{2}\right)
\end{array}\right),
\end{aligned}
$$

where

$$
\begin{aligned}
& A_{0}=\left.\frac{\partial f}{\partial x(t)}\right|_{x^{*}}=\left[\begin{array}{cc}
-k_{1} & 0 \\
0 & -k_{2}
\end{array}\right], \\
& A_{1}=\left.\frac{\partial f}{\partial x\left(t-\tau_{1}\right)}\right|_{x^{*}}=\left[\begin{array}{cc}
0 & a_{1} \\
0 & 0
\end{array}\right], \\
& A_{2}=\left.\frac{\partial f}{\partial x\left(t-\tau_{2}\right)}\right|_{x^{*}}=\left[\begin{array}{cc}
0 & 0 \\
\frac{-a_{2}}{\left(1+x_{1 e}\right)^{2}} & 0
\end{array}\right] .
\end{aligned}
$$

The system (7) is stable for $\tau_{1}=\tau_{2}=\infty$ since $A_{0}$ is Hurwitz for all $k_{1}, k_{2}>0$. The system is also stable without a delay $\left(\tau_{1}=\tau_{2}=0\right)$ since $\left(A_{0}+A_{1}+A_{2}\right)$ is Hurwitz for all $k_{1}, k_{2}, a_{1}, a_{2}>0$. We then have the following result.

Proposition 1. If there exist symmetric matrices $P>$ $0, S_{1}>0, S_{2}>0$ such that the following LMI holds:

$$
R=\left[\begin{array}{ccc}
P A_{0}+A_{0}^{\top} P+\sum_{i=1}^{2} S_{i} & P A_{1} & P A_{2} \\
A_{1}^{\top} P & -S_{1} & 0 \\
A_{2}^{\top} P & 0 & -S_{2}
\end{array}\right]<0,
$$

then the system (7) is asymptotically stable, independent of the delay.
Proof. The proof here constitues a direct consequence of Proposition 7.1 by $\mathrm{Gu}$ et al. (2003) and is obtained by considering a Lyapunov-Krasovskii functional that satisfies (5),

$$
\begin{aligned}
V(\phi)= & \phi^{\top}(0) P \phi(0) \\
& +\sum_{i=1}^{2} \int_{-\tau_{i}}^{0} \phi^{\top}(\theta) S_{i} \phi(\theta) \mathrm{d} \theta .
\end{aligned}
$$

Differentiating (9) along the system trajectories (7), we obtain (8). We use the system parameters (Loiseau et al., 2009) $k_{1}=0.03, k_{2}=0.03, a_{1}=1, a_{2}=1$. Since the system (3) evolves on $\mathbb{R}_{+} \times \mathbb{R}_{+}$, the equilibrium point of interest is $\left(x_{1}^{*}, x_{2}^{*}\right)=(32.8371,0.9851)$. Numerically solving the LMI conditions (8) using the YALMIP Toolbox in Matlab, the matrices $P, S_{1}$ and $S_{2}$ were found to be

$$
\begin{aligned}
P & =\left[\begin{array}{cc}
0.0127 & 0 \\
0 & 10.7791
\end{array}\right], \\
S_{1} & =\left[\begin{array}{cc}
0.0002 & 1 \\
0 & 0.5014
\end{array}\right], \\
S_{2} & =\left[\begin{array}{cc}
0.0002 & 0 \\
0 & 0.0589
\end{array}\right] .
\end{aligned}
$$

The eigenvalues of $R$ are $(-0.0589,-0.0002$, $-0.0001,-0.0001)$ and hence sufficient conditions for positive definiteness of $V$ and negative definiteness of $\dot{V}$ are satisfied. Thus, the linearised system (7) is locally asymptotically stable.

2.4. Nonlinear delay-independent stability analysis. We first introduce a change of coordinates $z_{1}=x_{1}$ $x_{1}^{*}, z_{2}=x_{2}-x_{2}^{*}$; the origin $\left(z_{1}=0, z_{2}=0\right)$ is the unique equilibrium point of interest. $z_{1}=x_{1}-x_{1}^{*}, z_{2}=x_{2}-x_{2}^{*}$. The system (3) in the new coordinates is

$$
\begin{aligned}
& \dot{z}_{1}(t)=-k_{1} z_{1}(t)+a_{1} z_{2}\left(t-\tau_{1}\right)+\left(a_{1} x_{2}^{*}-k_{1} x_{1}^{*}\right), \\
& \dot{z}_{2}(t)=-k_{2} z_{2}(t)+\frac{a_{2}}{\left(1+x_{1}^{*}\right)+z_{1}\left(t-\tau_{2}\right)} .
\end{aligned}
$$

We next analyze the delay-independent stability of the origin $z=0$.

Proposition 2. The origin $z=0$ of 10 is asymptotically delay-independent stable.

Proof. The construction of an appropriate L-K functional proves the stability of the equilibrium point, but the search for such a functional form is involved. The technique of the SOS simplifies the task by recasting the Lyapunov conditions in the form of LMI conditions, thereafter the SOS conditions can be verified in polynomial time by solving an appropriate semi-definite program. We apply 
the delay-independent stability result for the SOS given by Loiseau et al. (2009). The SOS technique is used to construct a Lyapunov-Krasovskii functional of the form

$$
V(\phi)=v_{0}(\phi(0))+\sum_{i=1}^{2} \int_{-\tau_{i}}^{0} v_{1 i}(\phi(\theta)) \mathrm{d} \theta .
$$

The sufficient conditions in Proposition 1 of Loiseau et al. (2009) are used to prove delay-independent asymptotic stability. SOS polynomials are used to construct the kernel in the Lyapunov-Krasovskii functional (11). The following choice of SOS polynomials is given as inputs to the SOSTOOLS Toolbox of Matlab,

$$
\begin{aligned}
\phi(z) & =z_{1}^{2}+z_{2}^{2}, \\
\psi(z) & =d_{1} z_{1}^{2}+d_{2} z_{2}^{2}, \\
v_{11} & =a_{1}\left(z_{1}^{2}+z_{2}^{2}\right)+a_{2}\left(z_{3}^{2}+z_{4}^{2}\right), \\
v_{12} & =a_{3}\left(z_{1}^{4}+z_{2}^{2}\right)+a_{4}\left(z_{5}^{4}+z_{6}^{2}\right),
\end{aligned}
$$

where $z_{3}=x_{1}\left(t-\tau_{1}\right), z_{4}=x_{2}\left(t-\tau_{1}\right), z_{5}=x_{1}\left(t-\tau_{2}\right)$, $z_{6}=x_{2}\left(t-\tau_{2}\right)$. Solving it, we obtained the following:

(i) $v_{0}$ has a Gram matrix decomposition $v_{0}=$ $Y(z)^{\top} Q Y(z)$, where $Y(z)$ is the vector of monomials, $Y(z)=\left(z_{2}, z_{2}^{2}, z_{1}, z_{1} z_{2}, z_{1}^{2}\right)$ and $Q>0$ is given by

$$
\left[\begin{array}{ccc}
49.5890 & -0.0015 & 0.0116 \\
-0.0015 & 0.0005 & 0 \\
0.0116 & 0 & 2.0707 \\
0 & 0 & -0.0001 \\
0.0001 & -0.0001 & 0 \\
& 0 & 0.0001 \\
& 0 & -0.0001 \\
-0.0001 & 0 \\
0.0002 & 0 \\
0 & 0
\end{array}\right] .
$$

(ii) $v_{11}$ and $v_{12}$ are given by

$$
\begin{aligned}
& v_{11}=437.84\left(z_{1}^{2}+z_{2}^{2}\right)+561.29\left(z_{3}^{2}+z_{4}^{2}\right), \\
& v_{12}=627.88\left(z_{1}^{4}+z_{2}^{2}\right)+437.34\left(z_{5}^{4}+z_{6}^{2}\right) .
\end{aligned}
$$

(iii) We next show that the derivative of $V\left(z_{t}\right)$ along the trajectories of 10 is locally negative definite. The Hessian of $\dot{V}\left(z_{t}\right)$, denoted by $\nabla_{z}^{2} \dot{V}\left(z_{t}\right)$, when evaluated at the origin is

$$
\left[\begin{array}{ccc}
-616.7679 & -0.04721 & 0 \\
-0.04722 & -874.0937 & 0 \\
0 & 0 & -875.68 \\
140.1329 & 0.7869 & 0 \\
-0.00074 & -2.931 & 0 \\
0 & 0 & 0
\end{array}\right.
$$

$\left.\begin{array}{ccc}140.1329 & -0.00074 & 0 \\ 0.7869 & -2.931 & 0 \\ 0 & 0 & 0 \\ -1122.58 & 0 & 0 \\ 0 & -1255.6 & 0 \\ 0 & 0 & -874.68\end{array}\right]$.

The coefficients $d_{1}$ and $d_{2}$ were found to be 1370 and 1335 , respectively. The eigenvalues of $\left.\nabla_{z}^{2} \dot{V}\left(z_{t}\right)\right|_{z=0}$ are $-1255.7825,-1158.81,-875.68,-874.68,-874.069$ and -580.5395 . Thus $\dot{V}$ is locally negative definite in an open set containing the origin and the result holds.

2.5. Simulations. In this section, we present plots showing the evolution of system trajectories described by (2) as the delays $\tau_{1}, \tau_{2}$ are varied. The parameters are fixed (Loiseau et al., 2009) to be $k_{1}=0.03, k_{2}=$ 0.03, $a_{1}=1, a_{2}=1$ and the initial conditions are $\left(z_{1}(0)=1, z_{2}(0)=1\right)$. Figure 3 depicts the trajectories approaching the equilibrium point when $\tau_{1}=0.01, \tau_{2}=$ 0.05, while Fig. 4 depicts the case when the delays are large with $\tau_{1}=10, \tau_{2}=5$. On the microscopic level, since delays cannot be in the order of seconds, the simulations show that the system is stable irrespective of the size of the delay.

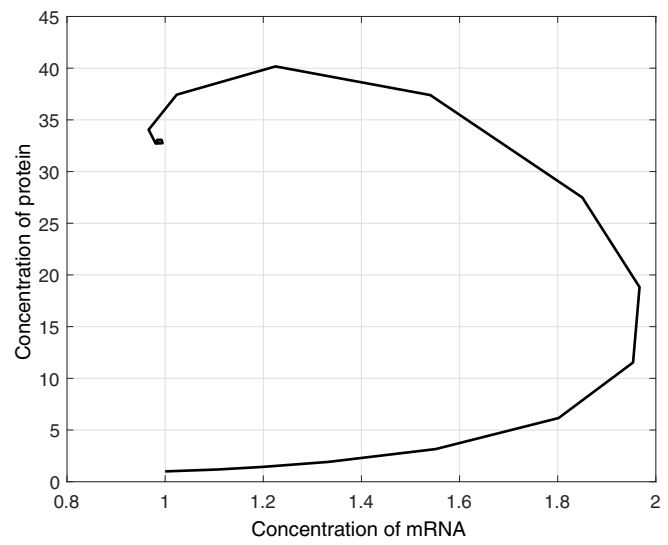

Fig. 3. Evolution of system trajectories with $\tau_{1}=0.01, \tau_{2}=$ 0.05

\section{Cancer immunotherapy model}

Cancer was the leading cause of death that accounted for around 8.2 million deaths worldwide in 2012 according to the WHO (World Health Organization). This number is expected to increase threefold by 2030. Traditional treatment methodologies for all types of cancer have been chemotherapy and radiation therapy, which are found effective in most cases. But they have major disadvantages, too. The prime one is that, even healthy cells are killed along with cancerous ones, and this has 


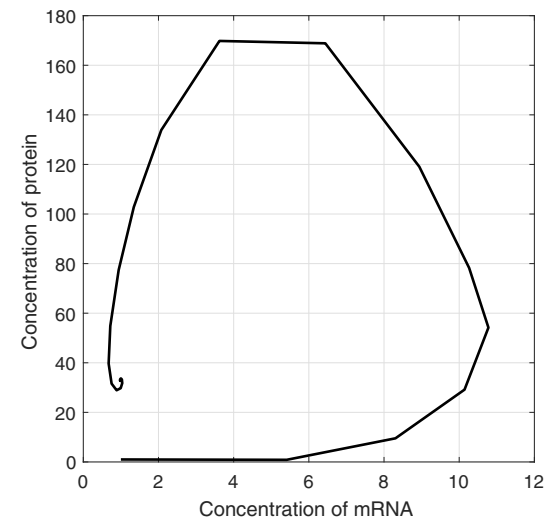

Fig. 4. Evolution of system trajectories with $\tau_{1}=10, \tau_{2}=5$.

many side effects. Also the protection offered by the treatment ends once it is stopped. New modalities of cancer treatment are now being sought after, which target cancerous cells specifically, overcoming the pitfalls of chemotherapy and radiation therapy. Immunotherapy is one such treatment method where the immune system is strengthened to fight against cancer. A brief idea of how the immune cells are triggered, finally leading to destruction of cancerous cells, is depicted in Fig. [5.

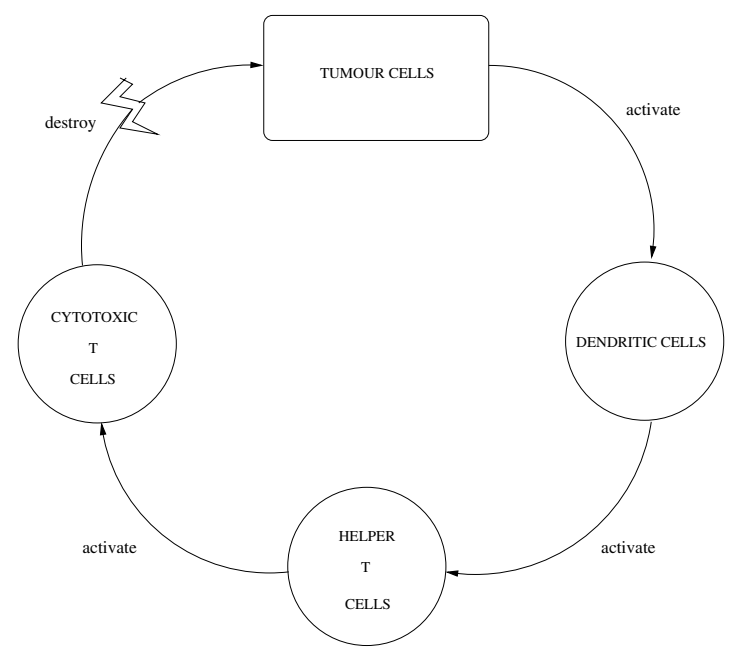

Fig. 5. Tumour destruction process.

The tumor-immune-cell interaction is an interesting phenomenon which is nonlinear and time-varying by nature. This nonlinearity makes the behaviour of tumors unpredictable and also appears to be one of the leading causes of death despite large measures being taken for its prevention and cure (d'Onofrio, 2008). A mathematical model of the Lotka-Volterra type (Melief, 2005; d'Onofrio, 2005) which captures the nonlinearity of tumor-immune-cell interactions is adopted and further studied in this paper. Predator-prey equations have accommodated this problem well (Bell, 1973) and have been a subject of active investigation (Andrew et al., 2007; Zhivkov and Waniewski, 2003; Banerjee, 2008).

To elaborate on this, the tumour cells contribute to prey population and helper $\mathrm{T}$ cells, cytotoxic $\mathrm{T}$ cells become the predators. A third-order predator-prey model with time-invariant parameters that portrays the phenomenon fairly accurately is studied in this paper. Higher-order differential equations can give better insight into the working of the system; however, this would require a large set of parameters and its estimation is an onerous task (Babbs, 2011). Hence we restrict our analysis to a third-order differential equation, which also includes a delay. This delay is an inevitable part of the process and physically implies the time taken for the activation of cytotoxic $\mathrm{T}$ cells or killer $\mathrm{T}$ cells. Incorporating it in the model helps study the dynamics in detail. The utility of control-theoretic tools in analyzing the models that capture tumor-immune-cell interactions lies in gaining better insight into immunotherapy and predicting the success or failure of treatment (Babbs, 2011). Stability analysis of the dynamics is critical to know whether the system trajectories converge to an equilibrium point or a limit cycle eventually, which gives information regarding the long-term behaviour and thus the outcome of therapy (Villasana and Radunskaya, 2003). In the work of Wang et al. (2012), a delayed oncolytic virus dynamics with continuous control is investigated and shown to exhibit Hopf bifurcation when the delay is increased beyond a certain limit.

3.1. Mathematical model. Consider the dynamics of immunotherapy which can be represented by the following predator-prey model (Saleem and Agrawal, 2012):

$$
\begin{aligned}
& \dot{x}_{1}(t)=1+a_{1} x_{1}\left(1-x_{1}\right)-k_{1} x_{1} x_{2}-k_{2} x_{1} \\
& \dot{x}_{2}(t)=a_{2} x_{2}(t-\tau) x_{3}(t-\tau)-a_{3} x_{2}-k_{3} x_{1} x_{2}, \\
& \dot{x}_{3}(t)=a_{4} x_{3}\left(1-x_{3}\right)-a_{5} x_{2} x_{3}-a_{6} x_{3}-k_{4} x_{1} x_{3}
\end{aligned}
$$

where

$$
\begin{aligned}
x_{1}= & \text { density of tumour cells, } \\
x_{2}= & \text { density of cytotoxic T cells, } \\
x_{3}= & \text { density of T helper cells, } \\
a_{1}= & \text { growth rate of tumour cells, } \\
a_{2}= & \text { delayed conversion rate of T helper cells to } \\
& \text { cytotoxic T cells, } \\
a_{3}= & \text { loss rate of cytotoxic T cells, } \\
a_{4}= & \text { growth rate of T helper cells, } \\
a_{5}= & \text { rate of decrease in T helper cells due to conversion, }
\end{aligned}
$$


$a_{6}=$ loss rate of T helper cells,

$k_{1}=$ rate of killing tumour cells by cytotoxic T cells,

$k_{2}=$ loss rate of tumour cells,

$k_{3}=$ rate of killing cytotoxic T cells by tumour cells,

$k_{4}=$ rate of killing $\mathrm{T}$ helper cells by tumour cells,

$\tau=$ delay in the activation of cytotoxic T cells,

$x_{i} \in \mathbb{R}_{+}, i=1,2,3, \tau \in \mathbb{R}_{++}$, and the normalized parameters $a_{1}, a_{2}, a_{3}, a_{4}, a_{5}, a_{6}, k_{1}, k_{2}, k_{3}, k_{4} \in \mathbb{R}_{++}$.

A starting point for the analysis would be to find the equilibrium points for the system described by (12). Physically, this implies an unstable steady state in which the tumour neither grows nor shrinks in size, which marks the initial point for theoretical explorations of strategies and effects (Babbs, 2011). Three equilibrium points for the dynamics described by (12) were obtained and are enumerated as follows:

$$
\begin{gathered}
E_{1}=\left\{x: x_{1}=s, x_{2}=x_{3}=0\right\}, \\
E_{2}=\left\{x: x_{1}=s, x_{2}=0\right. \\
\left.x_{3}=1-\frac{a_{6}}{a_{4}}-\frac{k_{4} x_{1}}{a_{4}}\right\},
\end{gathered}
$$

where

$$
s=\frac{1}{2}\left(1-\frac{k_{2}}{a_{1}}+\sqrt{\left(1-\frac{k_{2}}{a_{1}}\right)^{2}+\frac{4}{a_{1}}}\right) .
$$

The equilibrium point $E_{2}$ exists if and only if $a_{4}>a_{6}+$ $k_{4} x_{1}$. Let the third equilibrium point be denoted as $E_{3}=$ $\left(x_{1}^{*}, x_{2}^{*}, x_{3}^{*}\right)$. From (12) we can obtain the relation

$$
\begin{aligned}
x_{3}^{*} & =\frac{a_{3}+k_{3} x_{1}^{*}}{a_{2}}, \\
x_{2}^{*} & =\frac{a_{4}\left(1-x_{3}^{*}\right)-a_{6}-k_{4} x_{1}^{*}}{a_{5}}, \\
1 & +a_{1} x_{1}^{*}\left(1-x_{1}^{*}\right)-k_{1} x_{1}^{*} x_{2}^{*}-k_{2} x_{1}^{*}=0 .
\end{aligned}
$$

Substituting for $x_{2}^{*}$ and $x_{3}^{*}$, we obtain a quadratic equation in $x_{1}^{*}$,

$$
\left(x_{1}^{*}\right)^{2}+\frac{a}{b} x_{1}^{*}-\frac{1}{b}=0
$$

where

$$
\begin{aligned}
& a=k_{2}+\frac{k_{1} a_{4}}{a_{5}}\left(1-\frac{a_{3}}{a_{2}}-\frac{a_{6}}{a_{4}}\right)-a_{1}, \\
& b=a_{1}-\frac{k_{1}}{a_{5}}\left(k_{4}+\frac{k_{3} a_{4}}{a_{2}}\right) .
\end{aligned}
$$

A unique positive root for (13) exists provided

$$
-\frac{a}{b}+\sqrt{\left(\frac{a}{b}\right)^{2}+\frac{4}{b}}>0
$$

3.2. Nature of equilibria. We analyze the nature of each of the equilibria of the system $E_{1}, E_{2}, E_{3}$ by linearizing around the respective equilibrium points. Linearizing (12) about $x_{e}=\left(x_{1 e}, x_{2 e}, x_{3 e}\right)$ and with $\tilde{x}=x-x_{e}$, we obtain the linearized system as

$$
\left(\begin{array}{c}
\dot{\tilde{x}}_{1}(t) \\
\dot{\tilde{x}}_{2}(t) \\
\dot{\tilde{x}}_{3}(t)
\end{array}\right)=A_{0}\left(\begin{array}{c}
\tilde{x}_{1}(t) \\
\tilde{x}_{2}(t) \\
\tilde{x}_{3}(t)
\end{array}\right)+A_{1}\left(\begin{array}{c}
\tilde{x}_{1}(t-\tau) \\
\tilde{x}_{2}(t-\tau) \\
\tilde{x}_{3}(t-\tau)
\end{array}\right),
$$

where

$$
\begin{aligned}
A_{0} & =\left[\begin{array}{ccc}
d_{1} & -k_{1} x_{1 e} & 0 \\
-k_{3} x_{2 e} & -a_{3}-k_{3} x_{1 e} & 0 \\
-k_{4} x_{3 e} & -a_{5} x_{3 e} & d_{2}
\end{array}\right], \\
A_{1} & =\left[\begin{array}{ccc}
0 & 0 & 0 \\
0 & a_{2} x_{3 e} & a_{2} x_{2 e} \\
0 & 0 & 0
\end{array}\right] .
\end{aligned}
$$

with $d_{1}=a_{1}-2 a_{1} x_{1 e}-k_{1} x_{2 e}-k_{2}$ and $d_{2}=$ $a_{4}-2 a_{4} x_{3 e}-a_{5} x_{2 e}-a_{6} k_{4} x_{1 e}$. We fix the normalized parameters (Saleem and Agrawal, 2012) that satisfy biologically feasible conditions as $a_{1}=2.5, a_{2}=4.5$, $a_{3}=0.6, a_{4}=3.5, a_{5}=2, a_{6}=0.1, k_{1}=1$, $k_{2}=0.01, k_{3}=0.05$ and $k_{4}=0.001$. The local stability properties of the equilibrium points using linear models are summarized as follows:

1. $E_{1}=(1.303,0,0)$ and the eigenvalues of the matrix $A_{0}+A_{1}$ are

$$
\{-4.0249,-0.6651,3.3987\} \text {. }
$$

Since one of the eigenvalues is positive, $E_{1}$ is unstable for a zero delay.

2. $E_{2}=(1.3030,0,0.9706)$ and the eigenvalues of the matrices $A_{0}$ and $A_{0}+A_{1}$ are

$$
\{-3.3952 \pm i 4.0249,-0.6651\}
$$

and

$$
\{-3.3952 \pm i 4.0249,3.7023\}
$$

respectively. $A_{0}+A_{1}$ has a positive eigenvalue and hence $E_{2}$ is unstable for zero delay.

3. $E_{3}=(0.8740,1.4492,0.1430)$ and the eigenvalues of the matrices $A_{0}$ and $A_{0}+A_{1}$ are

$$
\{-0.5007,-0.6203,-3.3525\}
$$

and

$$
\{-3.3449,-0.2424 \pm i 1.3373\} \text {, }
$$


respectively.

A necessary (but not sufficient) condition for a system to be stable under the presence of a delay is that it is stable for zero delays. Stability analysis via linearization shows that the equilibrium points $E_{1}, E_{2}$ are unstable for zero delays $(\tau=0)$ and therefore it would be unstable for nonzero delays. In the next section, we therefore analyze the nonlinear stability of $E_{3}$, which is the tumour-free equilibrium point.

3.3. Nonlinear stability analysis. With the change of coordinates defined by $z_{i}=x_{i}-x_{i}^{*}, i=1,2,3$, the origin is the equilibrium point of interest in the transformed system. The system (12) in the new coordinates is

$$
\begin{aligned}
\dot{z}_{1}= & 1-a_{1} z_{1}^{2}+C_{1} z_{1}-k_{1} z_{1} z_{2}-C_{2} z_{2}-C_{3}, \\
\dot{z}_{2}= & C_{4} z_{1}+C_{5} z_{2}-k_{3} z_{1} z_{2}+C_{6} z_{2}(t-\tau) \\
& +a_{2} z_{2}(t-\tau) z_{3}(t-\tau)+C_{8} \\
& +C_{7} z_{3}(t-\tau), \\
\dot{z}_{3}= & C_{9} z_{1}-k_{4} z_{1} z_{3}+C_{10} z_{2}-a_{5} z_{2} z_{3} \\
& +C_{11} z_{3}+C_{12},
\end{aligned}
$$

with the constants defined as

$$
\begin{aligned}
C_{1} & =a_{1}-2 a_{1} x_{1}^{*}-k_{1} x_{2}^{*}-k_{2}, \\
C_{2} & =k_{1} x_{1}^{*}, \\
C_{3} & =1+a_{1} x_{1}^{*}\left(1-x_{1}^{*}\right)-k_{1} x_{1}^{*} x_{2}^{*}-k_{2} x_{1}^{*}, \\
C_{4} & =-k_{3} x_{2}^{*}, \\
C_{5} & =-a_{3}-k_{3} x_{1}^{*}, \\
C_{6} & =a_{2} x_{3}^{*} \\
C_{7} & =a_{2} x_{2}^{*} \\
C_{8} & =a_{2} x_{2}^{*} x_{3}^{*}-a_{3} x_{2}^{*}-k_{3} x_{1}^{*} x_{2}^{*}, \\
C_{9} & =-k_{4} x_{3}^{*}, \\
C_{10} & =-a_{5} x_{3}^{*}, \\
C_{11} & =a_{4}-2 a_{4} x_{3}^{*}-a_{5} x_{2}^{*}-a_{6}-k_{4} x_{1}^{*}, \\
C_{12} & =a_{4} x_{3}^{*}-a_{4} x_{3}^{* 2}-a_{6} x_{3}^{*}-a_{5} x_{2}^{*} x_{3}^{*}-k_{4} x_{1}^{*} x_{3}^{*} .
\end{aligned}
$$

We analyze the delay-dependent stability of the origin $z=$ 0 . Since the system has two more equilibrium points, we restrict the stability region to the set

$$
\Omega=\left\{z_{t} \in \mathcal{C}\left([-\tau, 0], \mathbb{R}^{3}\right):\left\|z_{t}\right\|_{2} \leq \gamma\right\} .
$$

The value of $\gamma$ is based on the location of the other two equilibrium points. The state constraint $|z(t+\theta)| \leq \gamma$ for all $\theta$ leads to the conditions

$$
\left.h_{1 i}=\left(z_{i}(t)-\gamma\right)\left(z_{i}(t)+\gamma\right)\right) \leq 0 \text {, }
$$$$
i=1,2,3
$$

and

$$
\begin{aligned}
\left.h_{2 j}=\left(z_{j}(t-\tau)-\gamma\right)\left(z_{j}(t-\tau)+\gamma\right)\right) \leq 0, & \\
& j=2,3 .
\end{aligned}
$$

Proposition 3. The origin of the system (13) is locally uniformly asymptotically stable for all delays in $[0, \tau]$.

Proof. We shall apply here the delay-dependent stability result proposed by Papachristodoulou (2004, Proposition 9). The SOS technique is used to obtain a Lyapunov functional having the structure

$$
\begin{aligned}
V\left(x_{t}\right)= & V_{0}(x(t))+\int_{-\tau}^{0} V_{1}(\theta, x(t), x(t+\theta)) \mathrm{d} \theta \\
& +\int_{-\tau}^{0} \int_{t+\theta}^{t} V_{2}(x(\zeta)) \mathrm{d} \zeta \mathrm{d} \theta
\end{aligned}
$$

The conditions in Proposition 9 of Papachristodoulou (2004) are rewritten by including the state constraints for the system (13) as

1. $V_{0}(z(t))-\phi(z(t)) \geq 0$,

2. $V_{1}(\theta, z(t), z(t+\theta))+p(z(t)) h(\theta) \geq 0$,

3. $V_{2}(z(\zeta)) \geq 0$,

4. the requirement that

$$
\begin{aligned}
& \tau \frac{\partial V_{1}}{\partial z(t)} f(z)+\frac{\partial V_{0}}{\partial z(t)} f(z)-\tau \frac{\partial V_{1}}{\partial \theta}+\tau V_{2}(z(t)) \\
& \quad-\tau V_{2}(z(t+\theta))+V_{1}(0, z(t), z(t)) \\
& \quad-V_{1}(-\tau, z(t), z(t-\tau))-p(z(t)) h(\theta) \\
& \quad-\sum_{i=1}^{3} h_{1 i} q_{1 i}(z(t), z(t-\tau)) \\
& \quad-\sum_{j=2}^{3} h_{2 j} q_{2 j}(z(t), z(t-\tau)) \\
& \leq 0, \quad \forall \theta \in[-\tau, 0] .
\end{aligned}
$$

We use SOS polynomials to construct the kernel in the Lyapunov-Krasovskii functional (15). The state constraints (14) are appended to the derivative of $V$ using SOS multipliers $h_{i j}, i=1,2,3, j=2,3$, whose coefficients are unknown. The condition $\theta \in[-\tau, 0]$ can be included by defining $h(\theta)=\theta(\theta+\tau)$. Since the polynomial $V_{1}(\theta, z(t), z(t+\theta))$ is required to be non-negative only when $h(\theta)=\theta(\theta+\tau)$ is satisfied, using the SOS multiplier $p(z(t))$ with known coefficients is used to write the condition

$$
V_{1}(\theta, z(t), z(t+\theta))+p(z(t)) h(\theta) \geq 0 .
$$


Since the equilibrium points other than the origin are located at $E_{1}$ and $E_{2}$, we fix $\gamma=0.14$. With the following choice of SOS polynomials as inputs to the SOSTOOLS Toolbox of Matlab:

$$
\begin{aligned}
\tau= & 0.017, \\
V_{1}= & z_{1}^{2}+z_{2}^{2}+z_{3}^{2}+z_{2}^{2}(t-\tau)+z_{3}^{2}(t-\tau), \\
V_{2}= & 0, \\
\phi(z)= & z_{1}^{4}+z_{2}^{4}+z_{3}^{4}, \\
h(\theta)= & \theta(\theta+\tau), \\
p(z)= & z_{1}^{4}+z_{2}^{4}+z_{3}^{4}, \\
q_{i j}= & b_{1} z_{1}^{4}+b_{2} z_{2}^{4}+b_{3} z_{3}^{4}+b_{4} z_{2}^{4}(t-\tau) \\
& +b_{5} z_{3}^{4}(t-\tau), \quad i=1,2,3, j=2,3,
\end{aligned}
$$

we obtain $V_{0}$ to be positive definite with a Gram matrix decomposition $V_{0}=Y(z)^{\top} Q Y(z)$, where $Q \in \mathbb{R}^{9 \times 9}$ satisfies $Q=Q^{\top}>0$ and is given by Eqn. (16) while $Y(z)$ is the vector of monomials,

$$
Y(z)=\left(z_{3}, z_{3}^{2}, z_{2}, z_{2} z_{3}, z_{2}^{2}, z_{1}, z_{1} z_{3}, z_{1} z_{2}, z_{1}^{2}\right) .
$$

Further, SOS multipliers associated with the state constraints are obtained as

$$
\begin{aligned}
q_{11}= & 35.0677 z_{1}^{4}-120.7356 z_{2}^{4}+28.6153 z_{3}^{4} \\
& +13.4857 z_{2}^{4}(t-\tau)-5.2187 z_{3}^{4}(t-\tau), \\
q_{12}= & 60.676 z_{1}^{4}+201.2121 z_{2}^{4}+54.9288 z_{3}^{4} \\
& +63.9426 z_{2}^{4}(t-\tau)+65.9958 z_{3}^{4}(t-\tau), \\
q_{13}= & 33.0355 z_{1}^{4}-117.8368 z_{2}^{4} \\
& +23.2452 z_{3}^{4}+16.5974 z_{2}^{4}(t-\tau) \\
& -2.2783 z_{3}^{4}(t-\tau), \\
q_{22}= & 30.6538 z_{1}^{4}-91.3417 z_{2}^{4}+27.5387 z_{3}^{4} \\
& +39.0185 z_{2}^{4}(t-\tau)+10.7666 z_{3}^{4}(t-\tau), \\
q_{23}= & 29.1352 z_{1}^{4}+2.2585 z_{2}^{4}+26.9202 z_{3}^{4} \\
& +41.4196 z_{2}^{4}(t-\tau)+45.2304 z_{3}^{4}(t-\tau) .
\end{aligned}
$$

We next show that the derivative of $V\left(z_{t}\right)$ along the trajectories of (13) is locally negative definite. The Hessian of $\dot{V}\left(z_{t}\right)$, denoted by $\nabla_{z}^{2} \dot{V}\left(z_{t}\right)$, when evaluated at the origin, is

$$
-\left[\begin{array}{ccccc}
12.163 & 2.471 & -1.638 & -0.163 & -1.649 \\
2.471 & 0.554 & -0.269 & -0.058 & -0.586 \\
-1.638 & -0.269 & 0.330 & 0.003 & 0.035 \\
-0.163 & -0.058 & 0.003 & 2 & 0 \\
-1.649 & -0.586 & 0.035 & 0 & 2
\end{array}\right]
$$

The eigenvalues of $\left.\nabla_{z}^{2} \dot{V}\left(z_{t}\right)\right|_{z=0}$ are -0.000379 , $-0.103245,-1.789669,-2.000000$ and -13.154485 .
Thus $\dot{V}$ is locally negative definite in an open set containing the origin and the result holds.

3.4. Simulations. In this section we present plots showing the evolution of system trajectories described by (13) with a zero delay and as the delay $\tau$ is varied. The plots were obtained with parameters fixed as described in Section 3 and initial conditions for $\left(z_{1}(0)=0.1, z_{2}(0)=\right.$ $\left.0.1, z_{3}(0)=0.1\right)$. Figure 6 shows the system without a delay has the trajectories approaching the equilibrium point of interest $E_{3}$. Figure 7 depicts the trajectories approaching the equilibrium point with $\tau=0.30$. It was observed through simulations that for $\tau=0.40$ the system exhibits a limit cycle (Saleem and Agrawal, 2012), as depicted by Fig. 8. For larger delays the system tends to behave chaotically.

\section{Biological implications}

The advancements in technology have resulted in large amounts of data, mainly of qualitative nature. The challenge now lies in making sense of these data and developing methodologies to address important biological questions. Genetic regulatory networks are highly complex and hence it becomes necessary to design models describing their dynamical functioning. Protein and mRNA concentrations evolve with time and may converge towards some steady state, periodic behaviour, or other complex dynamical attractors. The emergence of these patterns from the dynamical interactions between the elements of the network and comparison with experimental data help scientists to solve important problems (Bernot et al., 2013). Also, with the advent of computer technology, the behaviour of a few dozen genes can be simulated, but it is tough when millions come into play. Characterising the qualitative behaviour helps in dealing with complexity and realizing such gene networks synthetically using integrated circuits like neurochips learnt from biological neural networks (Chen and Aihara, 2002). The goal of immunotherapy or

any other treatment for that matter is the eradication of the tumour. In the absence of therapy and given that the tumour is highly immunogenic, the system described by (12) has the tumour-free equilibrium point $E_{3}$ to be locally, uniformly, asymptotically stable over a certain delay interval. The biological relevance of this being, the eradication of the tumour may be achieved if the state of the system at the end of therapy belongs to the basin of attraction of the tumour-free equilibrium point, so that the system tends to this point eventually. This requires us to know the state of the system at the start of therapy accurately, which is a problem in clinical practice (d'Onofrio et al., 2010). With an increase in the delay, and for $\tau=0.4$, we observed through simulations that there is 


$$
Q=\left[\begin{array}{ccccccccc}
0.1507 & 0.0524 & -0.0027 & -0.0024 & 0.0029 & -0.2303 & -0.0011 & -0.0082 & -0.0328 \\
0.0524 & 28.4917 & -0.0114 & 0.9181 & -2.3018 & -0.1295 & -1.0589 & 0.1075 & 0.8676 \\
-0.0027 & -0.0114 & 0.0279 & -0.0018 & -0.0062 & 0.1264 & -0.0045 & 0.0023 & 0.0184 \\
-0.0024 & 0.9181 & -0.0018 & 4.0556 & 0.0263 & -0.0035 & 0.1638 & -0.1563 & 0.1900 \\
0.0029 & -2.3018 & -0.0062 & 0.0263 & 1.0738 & -0.0339 & 0.0594 & 0.2178 & -0.4999 \\
-0.2303 & -0.1295 & 0.1264 & -0.0035 & -0.0339 & 0.8936 & -0.0187 & 0.0127 & 0.1256 \\
-0.0011 & -1.0589 & -0.0045 & 0.1638 & 0.0594 & -0.0187 & 6.4606 & 0.2381 & -0.2664 \\
-0.0082 & 0.1075 & 0.0023 & -0.1563 & 0.2178 & 0.0127 & 0.2381 & 2.8670 & 0.3954 \\
-0.0328 & 0.8676 & 0.0184 & 0.1900 & -0.4999 & 0.1256 & -0.2664 & 0.3954 & 4.5046
\end{array}\right] .
$$

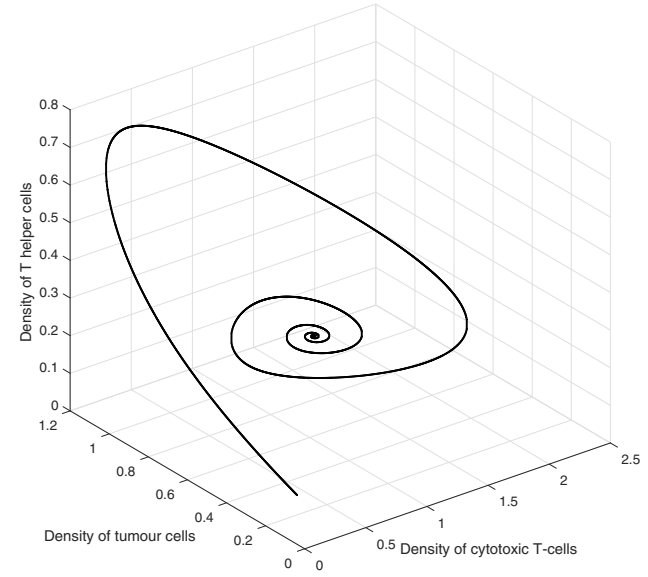

Fig. 6. Evolution of system trajectories with $\tau=0$.

an onset of periodic oscillations. The importance of such limit cycles is that the tumour levels oscillate around a fixed point without administering any therapy (Villasana and Radunskaya, 2003).

\section{Conclusions}

In this paper, we analysed the stability of a second-order model of a gene-regulatory network with delay using both linear and nonlinear approaches. A Lyapunov functional candidate having a similar structure for both linear and nonlinear approaches is constructed using LMIs in case of the former and SOSTOOLS in the case of the latter. The system is found to be stable independent of the delay. Through LMIs, sufficient conditions for local asymptotic stability of the equilibrium point were established. For the nonlinear system (10), a Lyapunov-Krasovskii functional of the form (11) with $v_{11}$ quadratic and $v_{0}, v_{12}$ quartic was obtained and delay-independent asymptotic stability of the origin was established.

For the third-order nonlinear model of immunotherapy for cancer, an L-K functional was constructed numerically to prove the delay-dependent stability of the system at a tumour-free equilibrium point. Since the system has multiple equilibria, only the local stability can be established. A Lyapunov functional of the

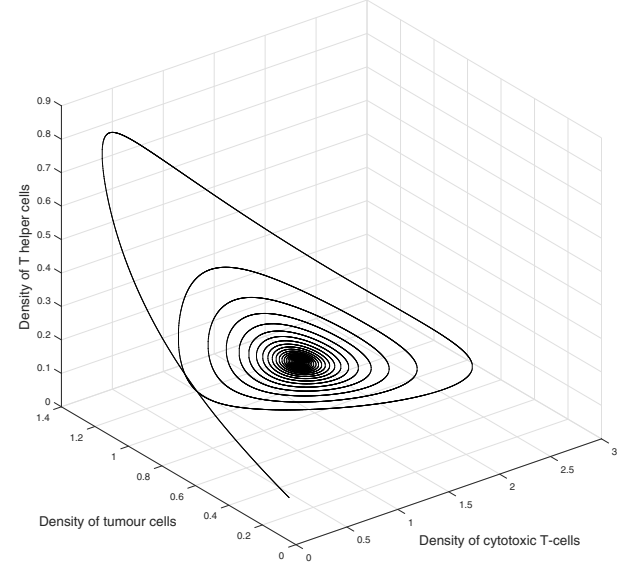

Fig. 7. Evolution of system trajectories with $\tau=0.3$.

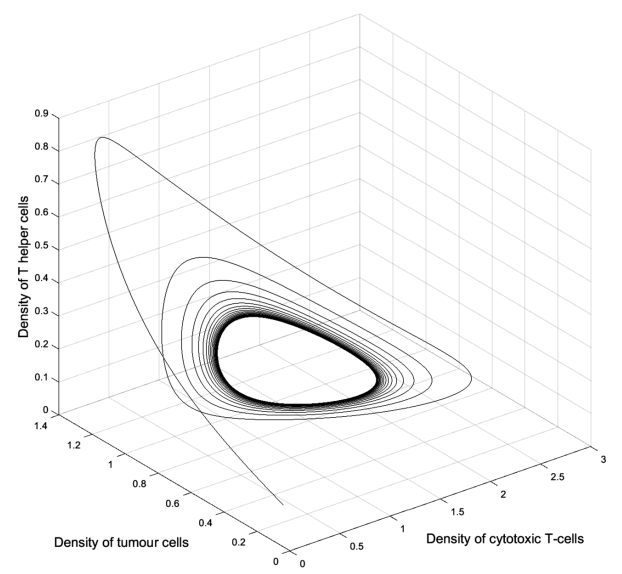

Fig. 8. Evolution of system trajectories with $\tau=0.4$.

form described by (15) with $V_{0}$ being quartic, $V_{1}$ being quadratic and $V_{2}=0$ was obtained, and thus delay dependent local uniform asymptotic stability of the origin in the delay interval $(0,0.017]$ is established. Through simulations we could obtain the maximum delay before the system breaks into periodic oscillations as $\tau \simeq$ 0.35 (Saleem and Agrawal, 2012). The SOS approach we adopted gives a maximum delay of 0.017 seconds, which is conservative due to a difficulty in handling nonlinearities with a delay. 


\section{References}

Aluru, S. (2005). Handbook of Computational Molecular Biology, CRC Press, Boca Raton, FL.

Andrew, S.M., Baker, C.T. and Bocharov, G.A. (2007). Rival approaches to mathematical modelling in immunology, Journal of Computational and Applied Mathematics 205(2): 669-686.

Babbs, C.F. (2011). Predicting success or failure of immunotherapy for cancer: Insights from a clinically applicable mathematical model, American Journal of Cancer Research 2(2): 204-213.

Banerjee, S. (2008). Immunotherapy with interleukin-2: A study based on mathematical modeling, International Journal of Applied Mathematics and Computer Science 18(3): 389-398, DOI: 10.2478/v10006-008-0035-6.

Bell, G.I. (1973). Predator-prey equations simulating an immune response, Mathematical Biosciences 16(3): 291-314.

Bernot, G., Comet, J.-P., Richard, A., Chaves, M., Gouzé, J.-L. and Dayan, F. (2013). Modeling and analysis of gene regulatory networks, in F. Cazals and P. Kornprobst (Eds.), Modeling in Computational Biology and Biomedicine: A Multidisciplinary Endeavor, Springer, Berlin/Heidelberg, pp. 47-80.

Bo, W., Yang, L. and Jianquan, L. (2012). New results on global exponential stability for impulsive cellular neural networks with any bounded time-varying delays, Mathematical and Computer Modelling 55(3): 837-843.

Bodnar, M. (2015). General model of a cascade of reactions with time delays: Global stability analysis, Journal of Differential Equations 259(2): 777-795.

Chen, L. and Aihara, K. (2002). Stability of genetic regulatory networks with time delay, IEEE Transactions on Circuits and Systems I: Fundamental Theory and Applications 49(5): 602-608.

De Jong, H. (2002). Modeling and simulation of genetic regulatory systems: A literature review, Journal of Computational Biology 9(1): 67-103.

d'Onofrio, A. (2005). A general framework for modeling tumor-immune-system competition and immunotherapy: Mathematical analysis and biomedical inferences, Physica D: Nonlinear Phenomena 208(3): 220-235.

d'Onofrio, A. (2008). Metamodeling tumor-immune-system interaction, tumor evasion and immunotherapy, Mathematical and Computer Modelling 47(5): 614-637.

d'Onofrio, A., Gatti, F., Cerrai, P. and Freschi, L. (2010). Delay-induced oscillatory dynamics of tumour-immune system interaction, Mathematical and Computer Modelling 51(5): 572-591.

Eduardo, L. and Ruiz-Herrera, A. (2013). Attractivity, multistability, and bifurcation in delayed Hopfield's model with non-monotonic feedback, Journal of Differential Equations 255(11): 4244-4266.

Goodwin, B.C. (1963). Temporal Organization in Cells: A Dynamic Theory of Cellular Control Processes, Academic Press, London/New York, NY.
Gu, K., Chen, J. and Kharitonov, V.L. (2003). Stability of TimeDelay Systems, Springer, New York, NY.

Kao, C.-Y. and Pasumarthy, R. (2012). Stability analysis of interconnected Hamiltonian systems under time delays, IET Control Theory and Applications 6(4): 570-577.

Kao, C.-Y. and Rantzer, A. (2007). Stability analysis of systems with uncertain time-varying delays, Automatica 43(6): 959-970.

Kauffman, S.A. (1969). Metabolic stability and epigenesis in randomly constructed genetic nets, Journal of Theoretical Biology 22(3): 437-467.

Kolmanovskii, V. and Myshkis, A. (1999). Introduction to the Theory and Applications of Functional Differential Equations, Springer, Dordrecht.

Liu, Y., Xu, P., Lu, J. and Liang, J. (2016a). Global stability of Clifford-valued recurrent neural networks with time delays, Nonlinear Dynamics 84(2): 767-777.

Liu, Y., Zhang, D., Lu, J. and Cao, J. (2016b). Global $\mu$-stability criteria for quaternion-valued neural networks with unbounded time-varying delays, Information Sciences 360: $273-288$.

Loiseau, J.J., Michiels, W., Niculescu, S.-I. and Sipahi, R. (2009). Topics in Time Delay Systems: Analysis, Algorithms and Control, Springer, Berlin/Heidelberg.

Mazenc, F. and Niculescu, S.-I. (2001). Lyapunov stability analysis for nonlinear delay systems, Systems \& Control Letters 42(4): 245-251.

Melief, C.J. (2005). Cancer immunology: Cat and mouse games, Nature 437(7055): 41-42.

Papachristodoulou, A. (2004). Analysis of nonlinear time-delay systems using the sum of squares decomposition, American Control Conference, Boston, MA, USA, pp. 4153-4158.

Papachristodoulou, A. and Prajna, S. (2005). A tutorial on sum of squares techniques for systems analysis, American Control Conference, Portland, OR, USA, pp. 2686-2700.

Parrilo, P.A. (2000). Structured Semidefinite Programs and Semialgebraic Geometry Methods in Robustness and Optimization, $\mathrm{PhD}$ thesis, California Institute of Technology, Pasadena, CA.

Pasumarthy, R. and Kao, C.-Y. (2009). On stability of time delay Hamiltonian systems, American Control Conference, St. Louis, MO, USA, pp. 4909-4914.

Richard, J.-P. (2003). Time-delay systems: An overview of some recent advances and open problems, Automatica 39(10): 1667-1694.

Saleem, M. and Agrawal, T. (2012). Complex dynamics in a mathematical model of tumor growth with time delays in the cell proliferation, International Journal of Scientific and Research Publications 2(6): 1-7.

Sharma, A., Kohar, V., Shrimali, M. and Sinha, S. (2014). Realizing logic gates with time-delayed synthetic genetic networks, Nonlinear Dynamics 76(1): 431-439. 
She, Z. and Li, H. (2013). Dynamics of a density-dependent stage-structured predator-prey system with beddingtondeangelis functional response, Journal of Mathematical Analysis and Applications 406(1): 188-202.

Thomas, R. (1991). Regulatory networks seen as asynchronous automata: A logical description, Journal of Theoretical Biology 153(1): 1-23.

Villasana, M. and Radunskaya, A. (2003). A delay differential equation model for tumor growth, Journal of Mathematical Biology 47(3): 270-294.

Wang, S., Wang, S. and Song, X. (2012). Hopf bifurcation analysis in a delayed oncolytic virus dynamics with continuous control, Nonlinear Dynamics 67(1): 629-640.

Yang, R., Wu, B. and Liu, Y. (2015). A Halanay-type inequality approach to the stability analysis of discrete-time neural networks with delays, Applied Mathematics and Computation 265: 696-707.

Zhivkov, P. and Waniewski, J. (2003). Modelling tumour-immunity interactions with different stimulation functions, International Journal of Applied Mathematics and Computer Science 13(3): 307-315.

Zhong, J., Lu, J., Liu, Y. and Cao, J. (2014). Synchronization in an array of output-coupled Boolean networks with time delay, IEEE Transactions on Neural Networks and Learning Systems 25(12): 2288-2294.

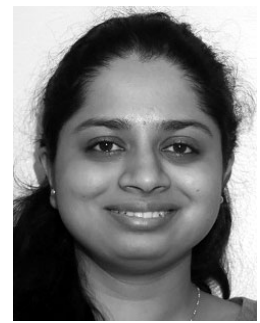

H.A. Kruthika received a Bachelor's degree in electronics and instrumentation from the BMS College of Engineering, affiliated to Visvesvaraya Technological University, in 2010. She then worked as a project associate at the Electronic Design Technology Laboratory, Indian Institute of Science, Bengaluru. She was granted a Master's degree by the Indian Institute of Technology Madras in 2016. Her research area covers mathematical modelling of biological systems.

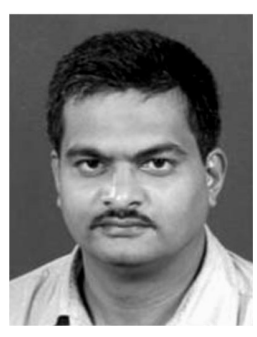

Arun D. Mahindrakar received the BEng degree in electrical and electronics from Karnatak University, Dharwad, India, in 1994, the MEng degree in control systems from VJTI, Mumbai, in 1997, and the PhD degree from IITB, Mumbai, in 2004. He is currently an associate professor in the Department of Electrical Engineering, IIT Madras, India. His research interests include nonlinear stability, geometric control and formation control of multi-agent systems.

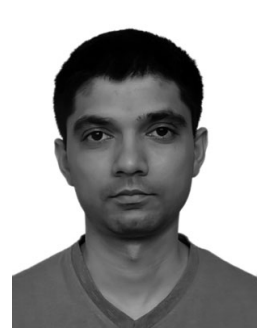

Ramkrishna Pasumarthy received the $\mathrm{PhD}$ degree in systems and control from the University of Twente, Enschede, The Netherlands, in 2006. $\mathrm{He}$ is currently an assistant professor with the Department of Electrical Engineering, IIT Madras, Chennai, India. His present research interests include the modeling and control of physical systems, infinite dimensional systems and control of computing systems, with a specific focus on cloud computing systems.

Received: 5 April 2016

Revised: 20 August 2016

Re-revised: 1 October 2016

Accepted: 12 October 2016 